\title{
Reflection Coefficient of a Waveguide with Slightly Uneven Walls
}

\author{
DAVID A. HILL, FELLOW, IEEE
}

\begin{abstract}
First-order results are derived for the reflection coefficient of a waveguide with slightly uneven walls. Specific analytical and numerical results are given for rectangular waveguides and coaxial transmission lines. Simple upper bounds are given for reflection coefficients in terms of the maximum deviation of the waveguide. For typical tolerances the reflection coefficients are very small $\left(<10^{-3}\right)$, but the results are important in precise six-port measurements.
\end{abstract}

\section{INTRODUCTION}

$\mathrm{N}$

ONUNIFORM waveguides have been studied for some time with application to antennas [1] and tapers [2]. The generalized telegrapher's equations [3], [4] provide a useful starting point, and Solymar [2] has worked with coupled traveling waves for studying spurious mode generation.

In this paper we use Solymar's formulation to derive first-order results for waveguides with small nonuniformity or wall roughness. Section II treats the reflection and transmission of the dominant mode and the generation of higher order modes for a waveguide of arbitrary cross section. Section III contains specific results for the reflection coefficient of the $\mathrm{TE}_{10}$ mode in a rectangular waveguide. Section IV contains similar results for the reflection coefficient of the TEM mode in a coaxial line. For typical tolerances the reflection coefficients are very small, but the results are important in precise six-port measurements [5].

\section{First-Order Solution}

We consider a perfectly conducting waveguide with a nonuniform section of length $L$ as shown in Fig. 1. Starting with the generalized telegrapher's equations of Reiter [4], Solymar has derived the following differential equations for coupled traveling waves [2]:

$$
\begin{aligned}
\frac{d A_{i}^{+}}{d z}= & -j \beta_{i} A_{i}^{+}-\frac{1}{2} \frac{d\left(\ln K_{i}\right)}{d z} A_{i}^{-} \\
& +\sum_{p}\left(S_{i p}^{+} A_{p}^{+}+S_{i p}^{-} A_{p}^{-}\right)
\end{aligned}
$$

and

$$
\begin{aligned}
\frac{d A_{i}^{-}}{d z}= & j \beta_{i} A_{i}^{-}-\frac{1}{2} \frac{d\left(\ln K_{i}\right)}{d z} A_{i}^{+} \\
& +\sum_{p}\left(S_{i p}^{-} A_{p}^{+}+S_{i p}^{+} A_{p}^{-}\right) .
\end{aligned}
$$

Manuscript received March 29, 1988; revised June 23, 1988.

The author is with the Electromagnetic Fields Division, National Bureau of Standards, Boulder, CO 80303.

IEEE Log Number 8824261 .

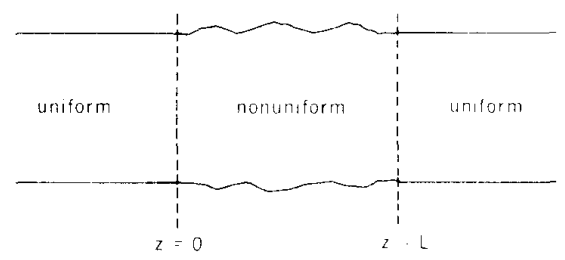

Fig. 1. Waveguide with a nonuniform section.

$A_{i}^{+}$and $A_{i}$ are amplitudes of the forward and backward traveling waves, subscript $i$ refers to the $i$ th mode, $\beta_{i}$ is the wavenumber of the $i$ th mode, $K_{i}$ is the wave impedance of the $i$ th mode, $S_{i p}^{+}$and $S_{i p}$ are forward and backward coupling coefficients, and the $p$ summations are over all waveguide modes. The time dependence is $\exp (j \omega t)$. In (1) it is assumed that $K_{i} \neq 0$ and $K_{i} \neq \infty$, and this means that $\beta_{i} \neq 0$. Thus (1) is not valid for modes at the cutoff frequency $\left(\beta_{i}=0\right)$. However, (1) remains valid for modes below cutoff where $\beta_{i}$ and $K_{i}$ are pure imaginary.

If the waveguide is fed by a single mode $m$, the boundary conditions at the ends of the nonuniform section are [2]

$$
A_{m}^{+}(0)=A_{0}, \quad A_{m}(L)=0
$$

and

$$
A_{i}^{+}(0)=0, \quad A_{i}(L)=0, \quad \text { for } i \neq m .
$$

Because we are interested in the effect of small waveguide roughness or imperfections, we assume that the waveguide cross section is nearly constant. Consequently we are able to use a perturbation solution. This is in contrast to the work of Solymar where intentional waveguide tapers were considered. The zero-order perturbation solution to (1) and (2) is the solution to the uniform guide, and only the forward-traveling $m$ th mode is nonzero:

$$
\begin{aligned}
& A_{m}^{+(0)}(z)=A_{0} e^{-j \beta_{m}^{(0)} z} \\
& A_{m}^{-(0)}(z)=0
\end{aligned}
$$

and

$$
A_{i}^{+(0)}(z)=A_{i}^{(0)}(z)=0, \quad \text { for } i \neq m \text {. }
$$

The superscript $(0)$ refers to zero-order quantities (uniform guide). 
A first-order analysis of (1) and (2) shows that the first-order amplitudes satisfy the following differential equations:

$$
\begin{aligned}
& \frac{d A_{m}^{+(1)}}{d z}=-j \beta_{m}^{(0)} A_{m}^{+(1)}-j\left(\beta_{m}^{(1)}-\beta_{m}^{(0)}\right) A_{m}^{+(0)} \\
& \frac{d A_{m}^{-(1)}}{d z}=j \beta_{m}^{(0)} A_{m}^{-(1)}+\left[S_{m m}^{-}-\frac{1}{2} \frac{d\left(\ln K_{m}\right)}{d z}\right] A_{m}^{+(0)} \\
& \frac{d A_{i}^{+(1)}}{d z}=-j \beta_{i}^{(0)} A_{i}^{+(1)}+S_{i m}^{+} A_{m}^{+(0)}, \quad i \neq m, \\
& \frac{d A_{i}^{-(1)}}{d z}=j \beta_{i}^{(0)} A_{i}^{-(1)}+S_{i m}^{-} A_{m}^{+(0)}, \quad i \neq m .
\end{aligned}
$$

Here the superscript (1) refers to first-order quantities. The solutions to the differential equations in (4) are

$$
\begin{aligned}
& A_{m}^{+(1)}(z)=A_{0} e^{-j \beta_{m}^{(0)} z}\left[1-j \int_{0}^{z}\left(\beta_{m}^{(1)}-\beta_{m}^{(0)}\right) d z^{\prime}\right] \\
& A_{m}^{-(1)}(z)=-A_{0} \int_{z}^{L}\left[S_{m m}^{-}-\frac{1}{2} \frac{d\left(\ln K_{m}\right)}{d z^{\prime}}\right] e^{-j \beta_{m}^{(0)}\left(z z^{\prime}-z\right)} d z^{\prime} \\
& A_{i}^{+(1)}(z)=A_{0} \int_{0}^{z} S_{i m}^{+} e^{-j\left[\beta_{m}^{(0)} z^{\prime}+\beta_{i}^{(0)}\left(z-z^{\prime}\right)\right]} d z^{\prime}, \quad i \neq m \\
& A_{i}^{-(1)}(z)=-A_{0} \int_{z}^{L} S_{i m}^{-} e^{-j\left[\beta_{m}^{(0)} z^{\prime}+\beta_{i}^{(0)}\left(z^{\prime}-z\right)\right]} d z^{\prime}, \quad i \neq m .
\end{aligned}
$$

We are actually most interested in the wave amplitudes at the ends of the nonuniform section; these are given by

$$
\begin{aligned}
& A_{m}^{+(1)}(L)=A_{0} e^{-j \beta_{m}^{(0)} L}\left[1-j \int_{0}^{L}\left(\beta_{m}^{(1)}-\beta_{m}^{(0)}\right) d z\right] \\
& A_{m}^{-(1)}(0)=-A_{0} \int_{0}^{L}\left[S_{m m}^{-}-\frac{1}{2} \frac{d\left(\ln K_{m}\right)}{d z}\right] e^{-j 2 \beta_{m}^{(0)} z} d z \\
& A_{i}^{+(1)}(L)=A_{0} \int_{0}^{L} S_{i m}^{+} e^{-j\left[\beta_{m}^{(0)} z+\beta_{i}^{(0)}(L-z)\right]} d z, \quad i \neq m \\
& A_{i}^{-(1)}(0)=-A_{0} \int_{0}^{L} S_{i m}^{-} e^{-j\left(\beta_{m}^{(0)}+\beta_{i}^{(0)}\right) z} d z, \quad i \neq m .
\end{aligned}
$$

The results in (6) are slightly different from those of Solymar, but they are equivalent to first order.

In our application only the $m$ th mode is propagating, and all other modes are below cutoff. Thus $\beta_{i}$ is negative imaginary for $i \neq m$, and $A_{i}^{+(1)}$ and $A_{i}^{-(1)}$ are negligible because of the exponential decay in the integrands (see Appendix A). The reflection coefficient $S_{11}$ and the transmission coefficient $S_{21}$ of the propagating mode $m$ are

$$
S_{11}=\frac{A_{m}^{-(1)}(0)}{A_{0}}=-\int_{0}^{L}\left[S_{m m}^{-}-\frac{1}{2} \frac{d\left(\ln K_{m}\right)}{d z}\right] e^{-j 2 \beta_{m}^{(0)} z} d z
$$

and

$$
S_{21}=\frac{A_{m}^{+(1)}(L)}{A_{0}}=e^{-j \beta_{m}^{(0)} L}\left[1-j \int_{0}^{L}\left(\beta_{m}^{(1)}-\beta_{m}^{(0)}\right) d z\right] .
$$

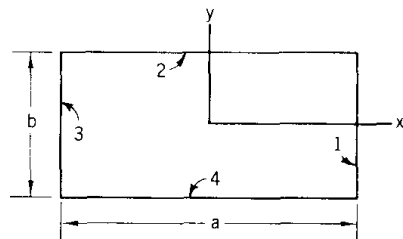

Fig. 2. Rectangular waveguide. The width $a$ and height $b$ are functions of $z$.

\section{Reflection COEFFICIENT FOR A RECTANGULAR WAVEGUIDE}

\section{A. Integral Form}

In this section we derive the reflection coefficient for the dominant $T E_{10}$ mode in a rectangular waveguide. The geometry for a rectangular waveguide of width $a$ and height $b$ is shown in Fig. 2. In our model $a$ and $b$ are allowed to vary with $z$, but the guide cross section is always rectangular. Using the notation of Solymar, we write the scalar mode function $\psi_{[10]}$ as

$$
\psi_{[10]}=\frac{1}{\pi}\left(\frac{2 a}{b}\right)^{1 / 2} \sin (\pi x / a)
$$

where [ ] on the subscript indicates a TE mode. The electric-field mode function $\boldsymbol{e}_{[10]}$ is [2]

$$
\boldsymbol{e}_{[10]}=\hat{z} \times \nabla_{t} \psi=\hat{\boldsymbol{y}}\left(\frac{2}{a b}\right)^{1 / 2} \cos (\pi x / a)
$$

where superscript ${ }^{\wedge}$ indicates a unit vector and $\nabla_{t}$ is the transverse gradient operator. The wavenumber $\beta_{[10]}$ is

$$
\beta_{[10]}=\left[k^{2}-(\pi / a)^{2}\right]^{1 / 2}
$$

where $k=2 \pi / \lambda$ and $\lambda$ is the wavelength in the medium filling the guide. The wave impedance $K_{[10]}$ is

$$
K_{[10]}=\frac{k(\mu / \epsilon)^{1 / 2}}{\beta_{[10]}}
$$

where $\mu$ and $\epsilon$ are the permeability and permittivity of the medium inside the guide.

The expression for the backward coupling coefficient $S_{[10 \| 10]}^{-}$is [2]

$$
S_{[10] 10]}=-\frac{1}{2} \oint_{C(z)} \tan \theta\left(\frac{\partial \psi_{[10]}}{\partial s}\right)^{2} d s
$$

where the integration is over the waveguide perimeter $C(z), d s$ is an element of $C(z)$, and $\tan \theta$ is the slope of the waveguide wall in the $z$ direction. The sidewalls ( 1 and 3 ) do not contribute to the integral in (12) because the $s$ derivative is zero. If we substitute (8) into (12) and perform some algebra, we can simply $S_{[10][10]}^{-\pi}$ to

$$
S_{[10\} 10]}^{-}=\frac{-1}{2 b} \frac{d b}{d z} .
$$

To evaluate (7), we also require the following derivative:

$$
\frac{d\left(\ln K_{[10]}\right)}{d z}=-a^{-1}\left[1-\left(\frac{\lambda}{2 a}\right)^{2}\right]^{-1}\left(\frac{\lambda}{2 a}\right)^{2} \frac{d a}{d z} \text {. }
$$


Equation (13) is consistent with Solymar's rectangular waveguide example, but he did not require (14) because he did not consider any variation in $a$.

In keeping with our first-order analysis, we write the guide width and height in the following form:

and

$$
a(z)=a_{0}+\Delta_{a}(z)
$$

$$
b(z)=b_{0}+\Delta_{b}(z)
$$

where $\Delta_{a} / a_{0} \ll 1, \Delta_{b} / b_{0} \ll 1$, and $a_{0}$ and $b_{0}$ are independent of $z$. In addition we require that $\Delta_{a}(0)=\Delta_{b}(0)=$ $\Delta_{a}(L)=\Delta_{b}(L)=0$ so that the waveguide surface is continuous. Using (13)-(15) in (7), we obtain the following first-order expression for $S_{11}$ :

$$
S_{11}=\int_{0}^{L}\left(C_{a} \frac{d \Delta_{a}}{d z}+C_{b} \frac{d \Delta_{b}}{d z}\right) e^{-j 2 \beta_{\left[1 b^{z}\right.}^{(0)}} d z
$$

where

$$
\begin{aligned}
& C_{a}=-\left\{2 a_{0}\left[1-\left(\frac{\lambda}{2 a_{0}}\right)^{2}\right]\right\}^{-1}\left(\frac{\lambda}{2 a_{0}}\right)^{2} \\
& C_{b}=\frac{1}{2 b_{0}}
\end{aligned}
$$

and

$$
\beta_{[10]}^{(0)}=\left[k^{2}-\left(\pi / a_{0}\right)^{2}\right]^{1 / 2}
$$

The expression in (16) provides a formal solution for $S_{11}$, but its form is inconvenient because it requires the derivatives of $\Delta_{a}$ and $\Delta_{b}$. Using integration by parts, we can rewrite (16) in the following form:

$$
S_{11}=S_{11 a}+S_{11 b}
$$

where

$$
S_{11 a}=j 2 \beta_{[10]}^{(0)} C_{a} \int_{0}^{L} \Delta_{a}(z) e^{-j 2 \beta_{[10]^{z}}^{(0)}} d z
$$

and

$$
S_{11 b}=j 2 \beta_{[10]}^{(0)} C_{b} \int_{0}^{L} \Delta_{b}(z) e^{-j 2 \beta_{[10]^{2}}^{(0)}} d z .
$$

This form is more convenient than (16) because it involves the width and height deviations, rather than their derivatives. We have broken $S_{11}$ into two parts to illustrate the separate dependencies on $\Delta_{a}$ and $\Delta_{b}$.

The form of (17) is similar to that in sea scatter [6] where the backscattered signal has the Bragg diffraction form. The integration in (17) essentially picks out the Fourier components of the surface variations with wavenumber $2 \beta_{[10]}^{(0)}$. If we are given the width and height variations, $\Delta_{a}(z)$ and $\Delta_{b}(z)$, we can calculate $S_{11}$ numerically from (17).

\section{B. Upper Bound}

Frequently the actual $z$ profiles of $\Delta_{a}$ and $\Delta_{b}$ are not known, but an estimate of the upper bound is available. Let us assume that

$$
\left|\Delta_{a}(z)\right| \leqslant \Delta_{\max }
$$

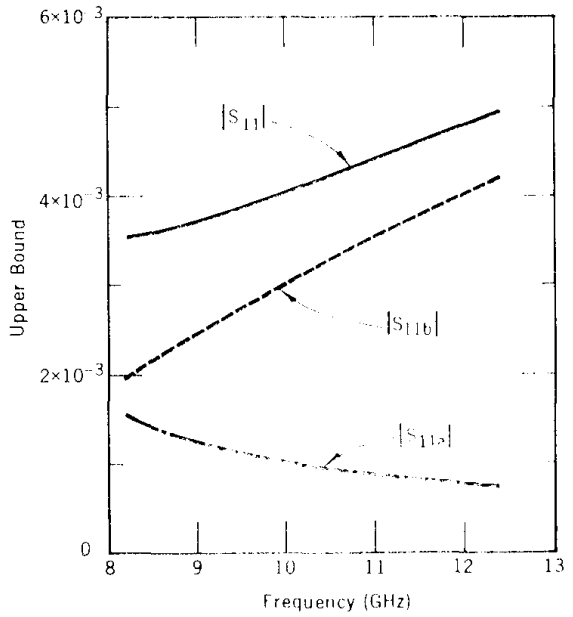

Fig. 3. Upper bound of the refiection coefficient magnitude $\left|S_{11}\right|$. Parameters: $a_{0}=2.29 \mathrm{~cm}, b_{0}=1.02 \mathrm{~cm}, l=7.62 \mathrm{~cm}$, and $\Delta_{\text {inax }}=2.54 \mu \mathrm{m}$.

and

$$
\left|\Delta_{b}(z)\right| \leqslant \Delta_{\max }
$$

where $\Delta_{\max }$ is a known dimensional tolerance. Then from (17), $\left|S_{11}\right|$ satisfies

$$
\left|S_{11}\right| \leqslant 2 \beta_{[10]}^{(0)} L\left(\left|C_{u}\right|+\left|C_{l}\right|\right) \Delta_{\text {max }} .
$$

Thus the upper bound on $\left|S_{11}\right|$ is directly proportional to $\Delta_{\max }$ and $L$. For realistic profiles of $\Delta_{a}$ and $\Delta_{b}$, the actual value of $\left|S_{11}\right|$ will normally be much smaller than the upper bound in (19) because of the oscillatory nature of the exponential factor in (17).

To illustrate the order of magnitude of the quantities in (19), we consider a six-port application at $X$ band. Typical parameters are [7]: $a_{0}=2.29 \mathrm{~cm}(0.9 \mathrm{in}), b_{0}=1.02 \mathrm{~cm}(0.4$ in), $L=7.62 \mathrm{~cm}(3 \mathrm{in}), \Delta_{\max }=2.54 \mu \mathrm{m}\left(10^{-4} \mathrm{in}\right)$. and frequency $=8.2-12.4 \mathrm{GHz}$. The upper bound on $\left|S_{11}\right|$ is shown in Fig. 3 as a function of frequency. Also shown are the separate contributions caused by variations in $a$ and b:

$$
\left|S_{11,2}\right| \leqslant 2 \beta_{\mid 1(1)\}}^{(1)} L \cdot\left|C_{d}^{\prime}\right| \Delta_{1) \text { mix }}
$$

and

$$
\left|S_{11 h}\right| \leqslant 2 \beta_{\{10\}}^{(0)} L,\left|C_{b}\right| \Delta_{\max } .
$$

The $\left|S_{11 a}\right|$ term can be viewed as an impedance effect and could be predicted by classical nonuniform transmission theory [8]. However, the $\left|S_{11 h}\right|$ term is caused by backward coupling into the same mode and is not predicted by a classical transmission line analysis. In general, the two terms are of the same order of magnitude. Their frequency dependence is different because $\left|C_{a l}\right|$ is frequency dependent and $\left|C_{b}\right|$ is not. In contrast the forward coupling coefficient is zero [2]. and changes in $b$ do not affect the transmission coefficient (sec Appendix B).

The treatment here has assumed that the nonuniform section of waveguide is continuous at the ends. $\Delta_{a}(0)=$ 
$\Delta_{b}(0)=\Delta_{a}(L)=\Delta_{b}(L)$. If there is a discontinuity at either end (as with a junction), then that effect must be addressed separately [9].

\section{Sinusoidal Profile}

In this section we consider an idealized sinusoidal profile for both $\Delta_{a}$ and $\Delta_{b}$. This is a convenient profile to consider because it is zero at both ends ( $z=a$ and $z=L)$ and the integrations in (17) can be performed analytically. The specific forms for $\Delta_{a}$ and $\Delta_{b}$ are

$$
\Delta_{a}(z)=\Delta_{a \max } \sin (n \pi z / L)
$$

and

$$
\Delta_{b}(z)=\Delta_{b \max } \sin (n \pi z / L)
$$

where $\Delta_{a \max }$ and $\Delta_{b \max }$ are maximum deviations and $n$ is a positive integer equal to the number of half cycles in the interval of length $L$.

To evaluate $S_{11}$ using (17), we need to evaluate the following integral:

$$
I_{n}=\int_{0}^{L} \sin (n \pi z / L) e^{-j 2 \beta_{[10]^{2} z}^{z}} d z .
$$

If we replace the sine factor by complex exponentials and perform some algebra, we can write $I_{n}$ as

$$
\begin{aligned}
I_{n}=\frac{L}{2 j}\left[e^{j\left(n \pi / 2-\beta_{[10]}^{(0)} L\right)} \frac{\sin \left(\frac{n \pi}{2}-\beta_{[10]}^{(0)} L\right)}{\left(\frac{n \pi}{2}-\beta_{[10]}^{(0)} L\right)}\right. \\
\\
\left.-e^{-j\left(n \pi / 2+\beta_{[10]}^{(0)} L\right)} \frac{\sin \left(\frac{n \pi}{2}+\beta_{[10]}^{(0)} L\right)}{\left(\frac{n \pi}{2}+\beta_{[10]}^{(0)} L\right)}\right] .
\end{aligned}
$$

The first term in (23) has a peak at $\beta_{[10]}^{(0)} L=n \pi / 2$, which is the condition for Bragg scatter.

Using (17) and (23), we can write $S_{11 a}$ and $S_{11 b}$ as

$$
S_{11 a}=j 2 \beta_{[10]}^{(0)} C_{a} \Delta_{a \max } I_{n}
$$

and

$$
S_{11 b}=j 2 \beta_{[10]}^{(0)} C_{b} \Delta_{b \max } I_{n} .
$$

Numerical results for the magnitudes of $S_{11 a}$ and $S_{11 b}$ are shown in Figs. 4 and 5 for $\Delta_{a \max }=\Delta_{b \max }=2.54 \mu \mathrm{m}$. The curves for $n=8$ have a peak in the center of the frequency band where the Bragg condition is satisfied. For $n=2$ and $n=20$, the Bragg condition is not satisfied, and the magnitudes are much lower. The results in Figs. 4 and 5 indicate that a special profile is required for the magnitudes to even approach the upper bounds, and generally the magnitudes are much lower.

\section{Reflection Coefficient for a CoAXIal Line}

\section{A. Integral Form}

In this section we derive the reflection coefficient for the dominant TEM mode in a coaxial line. The geometry for a coaxial line with inner radius $\rho_{i}$ and outer radius $\rho_{o}$ is

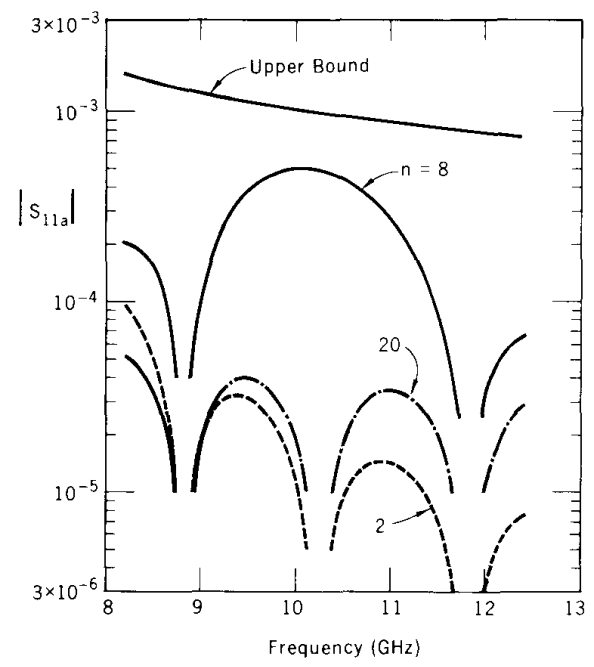

Fig. 4. Magnitude of $S_{11 a}$ for a sinusoidal profile. Parameters: $a_{0}=2.29$ $\mathrm{cm}, b_{0}=1.02 \mathrm{~cm}, L=7.62 \mathrm{~cm}$, and $\Delta_{a \max }=2.54 \mu \mathrm{m}$.

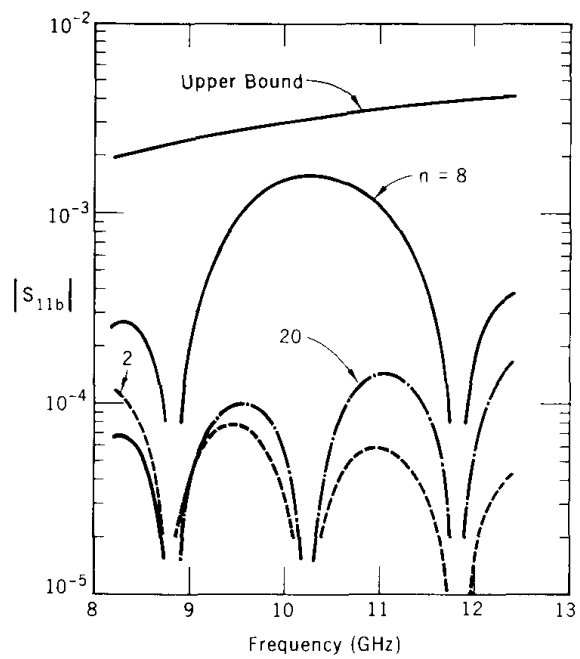

Fig. 5. Magnitude of $S_{11 b}$ for a sinusoidal profile. Parameters: $a_{0}=2.29$ $\mathrm{cm}, b_{0}=1.02 \mathrm{~cm}, L=7.62 \mathrm{~cm}$, and $\Delta_{b \text { max }}=2.54 \mu \mathrm{m}$.

shown in Fig. 6. In our model $\rho_{i}$ and $\rho_{0}$ are allowed to vary with $z$, but the guide cross section is always coaxial.

The electric-field mode function $e_{0}$ for the TEM mode is

$$
\boldsymbol{e}_{0}=\frac{\hat{\boldsymbol{\rho}}}{\left[2 \pi \ln \left(\rho_{o} / \rho_{i}\right)\right]^{1 / 2} \rho}
$$

where the subscript 0 indicates the TEM mode. The propagation constant $\beta_{0}$ of the TEM mode is the wavenumber of the medium:

$$
\beta_{0}=k=2 \pi / \lambda \text {. }
$$

The wave impedance $K_{0}$ of the TEM mode is the intrinsic impedance of the medium:

$$
K_{0}=\eta=(\mu / \epsilon)^{1 / 2} \text {. }
$$

Since $K_{0}$ does not depend on $\rho_{i}$ and $\rho_{o}$, the derivative 


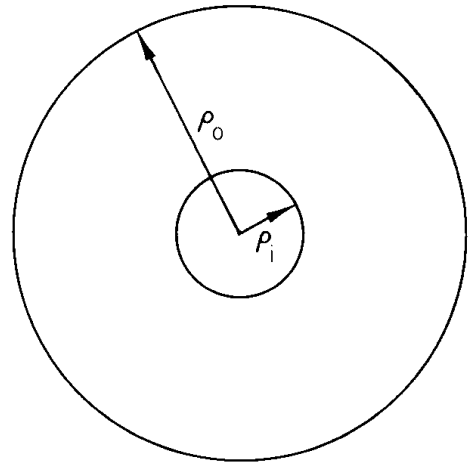

Fig. 6. Coaxial line. The inner and outer radii, $\rho_{i}$ and $\rho_{o}$, are functions of $z$.

term in (7) is zero. Thus the reflection coefficient $S_{11}$ of the TEM mode obtained from (7) is

$$
S_{11}=-\int_{0}^{L} S_{00}^{-} e^{-j 2 k z} d z
$$

The backward coupling coefficient $S_{00}^{-}$of the TEM mode is most directly determined from the general formula of Reiter [4] which is an integral over the cross section of the guide. For the coaxial line this integral takes the following form:

$$
S_{00}^{-}=\int_{0}^{2 \pi} \int_{\rho_{i}}^{\rho_{i}} \boldsymbol{e}_{0} \cdot \frac{d e_{0}}{d z} \rho d \rho d \phi .
$$

If we substitute (25) into (29) and carry out the integrations, we obtain

$$
S_{00}^{-}=\left[2 \ln \left(\rho_{o} / \rho_{i}\right)\right]^{-1}\left[\frac{1}{\rho_{i}} \frac{d \rho_{i}}{d z}-\frac{1}{\rho_{o}} \frac{d \rho_{o}}{d z}\right] .
$$

The result in (30) is equivalent to that of classical theory for nonuniform transmission lines [7] if we take into account the difference in the definition of voltage and current. In keeping with our first-order analysis, we write the inner and outer radii as

$$
\rho_{i}(z)=\rho_{i 0}+\Delta_{i}(z)
$$

and

$$
\rho_{o}(z)=\rho_{o 0}+\Delta_{o}(z)
$$

where $\Delta_{i} / \rho_{i 0} \ll 1, \Delta_{o} / \rho_{o 0} \ll 1$, and $\rho_{i 0}$ and $\rho_{o 0}$ are independent of $z$. Using (30) and (31) in (28), we obtain the following first-order solution for $S_{11}$ :

$$
S_{11}=\int_{0}^{L}\left(C_{i} \frac{d \Delta_{i}}{d z}+C_{o} \frac{d \Delta_{o}}{d z}\right) e^{-j 2 k z} d z
$$

where

$$
C_{i}=-\left[2 \rho_{i 0} \ln \left(\rho_{o 0} / \rho_{i 0}\right)\right]^{-1}
$$

and

$$
C_{o}=\left[2 \rho_{o 0} \ln \left(\rho_{o 0} / \rho_{i 0}\right)\right]^{-1} .
$$

As with the rectangular guide, we prefer not to deal with the derivatives of the dimensional variations. Using inte-

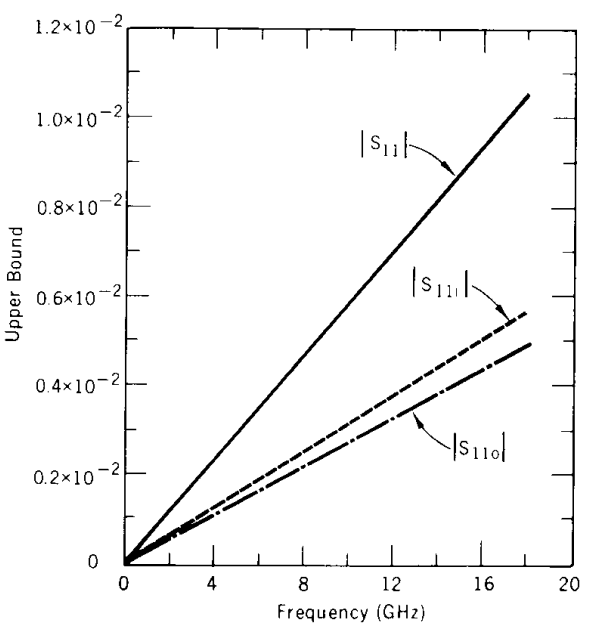

Fig. 7. Upper bound of the reflection coefficient $\left|S_{11}\right|$ for a coaxial line. Parameters: $\rho_{i}=1.52 \mathrm{~mm}, \rho_{o}=3.5 \mathrm{~mm}, L=3 \mathrm{~cm}, \Delta_{\text {i } \max }=0.635 \mu \mathrm{m}$, and $\Delta_{o \max }=1.27 \mu \mathrm{m}$.

gration by parts, we can rewrite (32) in the following form:

$$
S_{11}=S_{11 i}+S_{11 。}
$$

where

$$
S_{11 i}=j 2 k C_{i} \int_{0}^{L} \Delta_{i}(z) e^{-j 2 k z} d z
$$

and

$$
S_{11 o}=j 2 k C_{0} \int_{0}^{L} \Delta_{o}(z) e^{-j 2 k z} d z .
$$

We have again broken $S_{11}$ into two parts to illustrate the separate dependencies on $\Delta_{i}$ and $\Delta_{o}$.

\section{B. Upper Bound}

As with the rectangular waveguide, we can obtain an upper bound on $\left|S_{11}\right|$ if we have upper bounds on $\Delta_{i}$ and $\Delta_{o}$. Let us assume that

$$
\left|\Delta_{i}(z)\right| \leqslant \Delta_{i \max }
$$

and

$$
\left|\Delta_{o}(z)\right| \leqslant \Delta_{o \max }
$$

where $\Delta_{i \max }$ and $\Delta_{o \max }$ are known dimensional tolerances. Then, from (33), $\left|S_{11}\right|$ satisfies

$$
\left|S_{11}\right| \leqslant\left|S_{11 i}\right|+\left|S_{110}\right|
$$

where

$$
\left|S_{11 i}\right| \leqslant 2 k L\left|C_{i}\right| \Delta_{i \max }
$$

and

$$
\left|S_{11 o}\right| \leqslant 2 k L\left|C_{0}\right| \Delta_{o \max }
$$

For realistic profiles of $\Delta_{i}$ and $\Delta_{o}$, the actual value of $\left|S_{11}\right|$ will normally be much smaller than the upper bound in (35) because of the oscillatory nature of the exponential factor in (33).

To illustrate the order of magnitude of the quantities in (35), we consider a six-port application. Typical parame- 


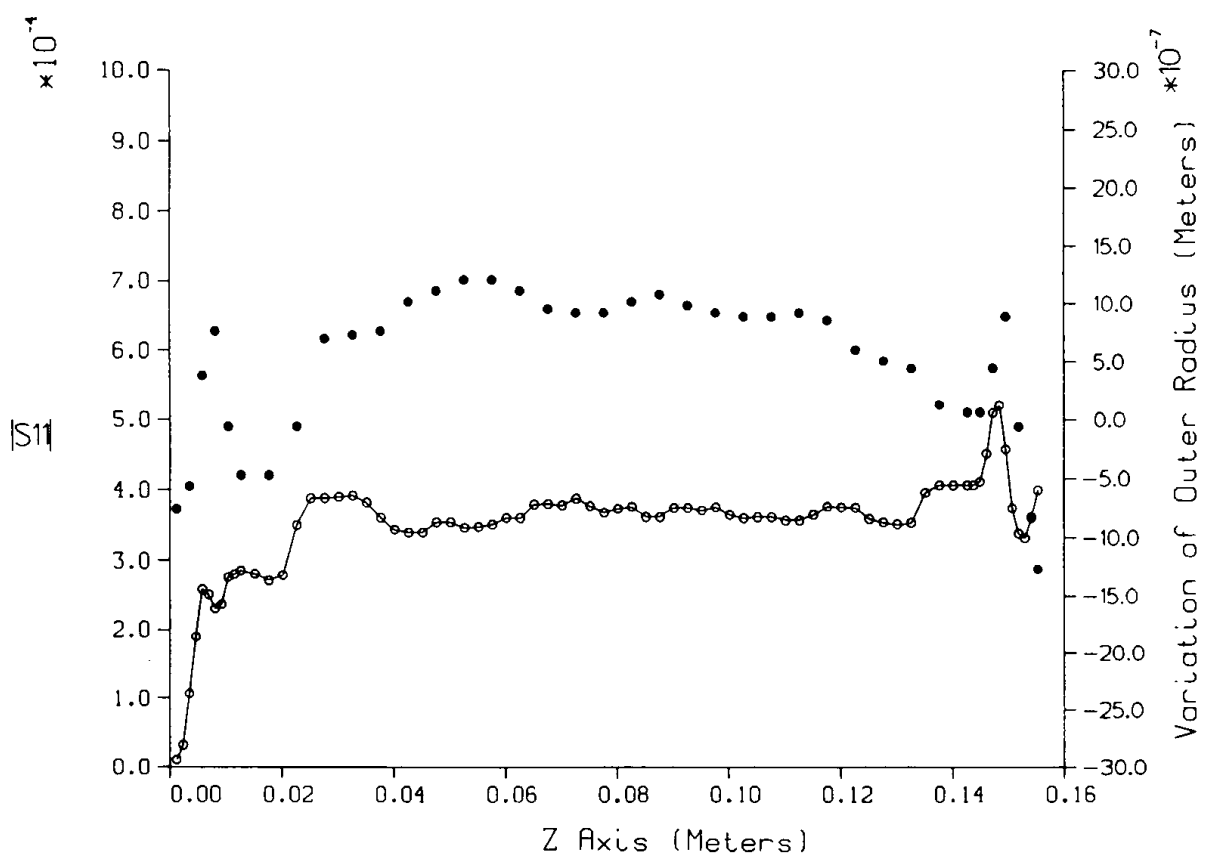

Fig. 8. Profile of the outer radius of a coaxial line and magnitude of the reflection coefficient for a frequency of $6 \mathrm{GHz}$ ( $7 \mathrm{~mm}$ air line).

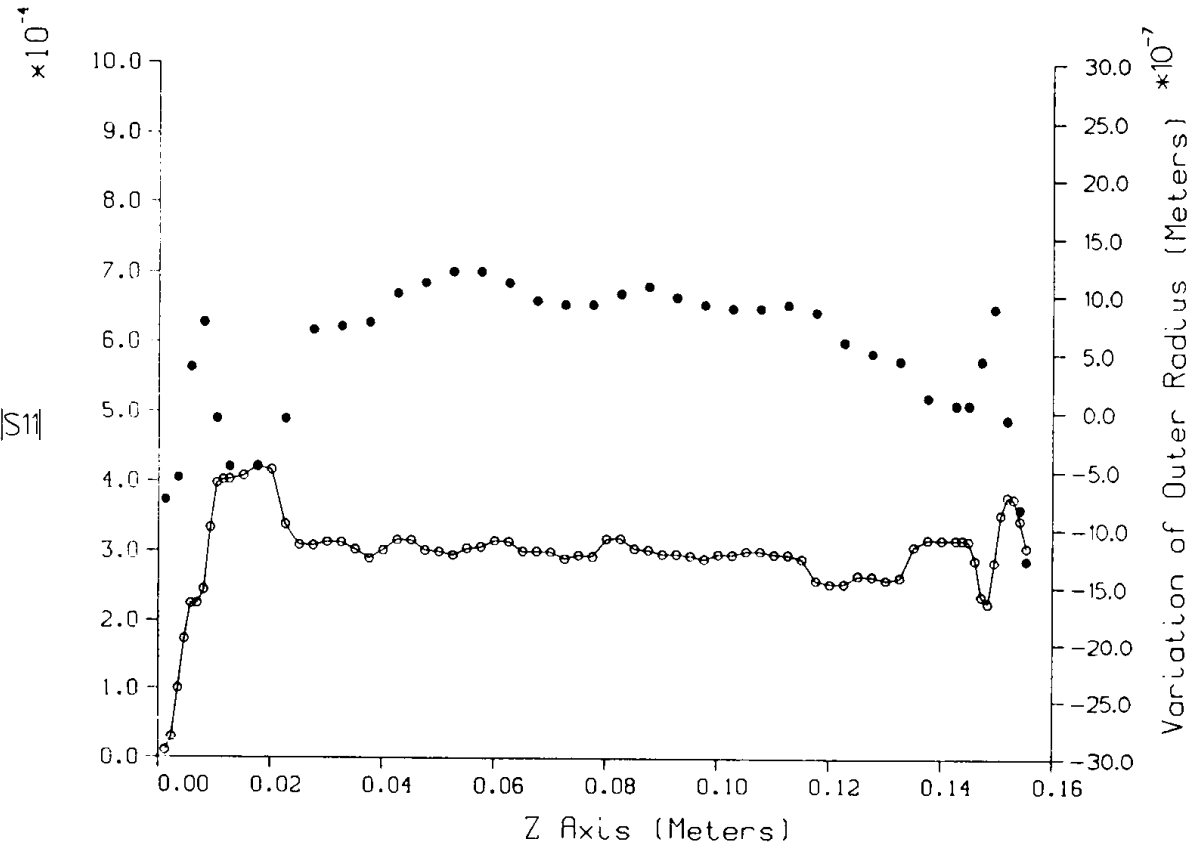

Fig. 9. Profile of the outer radius of a coaxial line and magnitude of the reflection coefficient for a frequency of $12 \mathrm{GHz}$ ( $7 \mathrm{~mm}$ air line).

ters are [7]: $\rho_{i}=1.52 \mathrm{~mm}, \rho_{o}=3.5 \mathrm{~mm}, L=3 \mathrm{~cm}, \Delta_{i \max }=$ $0.635 \mu \mathrm{m}, \Delta_{o \max }=1.27 \mu \mathrm{m}$, and frequency $\leqslant 18 \mathrm{GHz}$. The upper bounds on $\left|S_{11 i}\right|,\left|S_{110}\right|$, and $\left|S_{11}\right|$ are shown in Fig. 7. All three quantities are directly proportional to frequency because of the $k$ factor in (35). The results for a realistic profile would normally have a more complicated frequency dependence because of the $\exp (-j 2 k z)$ factor in the integrals in (33).

\section{Actual Profile}

The outer diameter of a precision air line was measured with an air gauge. The parameters are the same as in the previous case except that the line is longer $(L=16 \mathrm{~cm})$. We assume that the inner conductor has no variation $\left(\Delta_{i}=0\right)$. In Figs. 8-10, we show the magnitude of the reflection coefficient $\left|S_{11}\right|$ as a function of the length of the line for three different frequencies of interest. The actual 


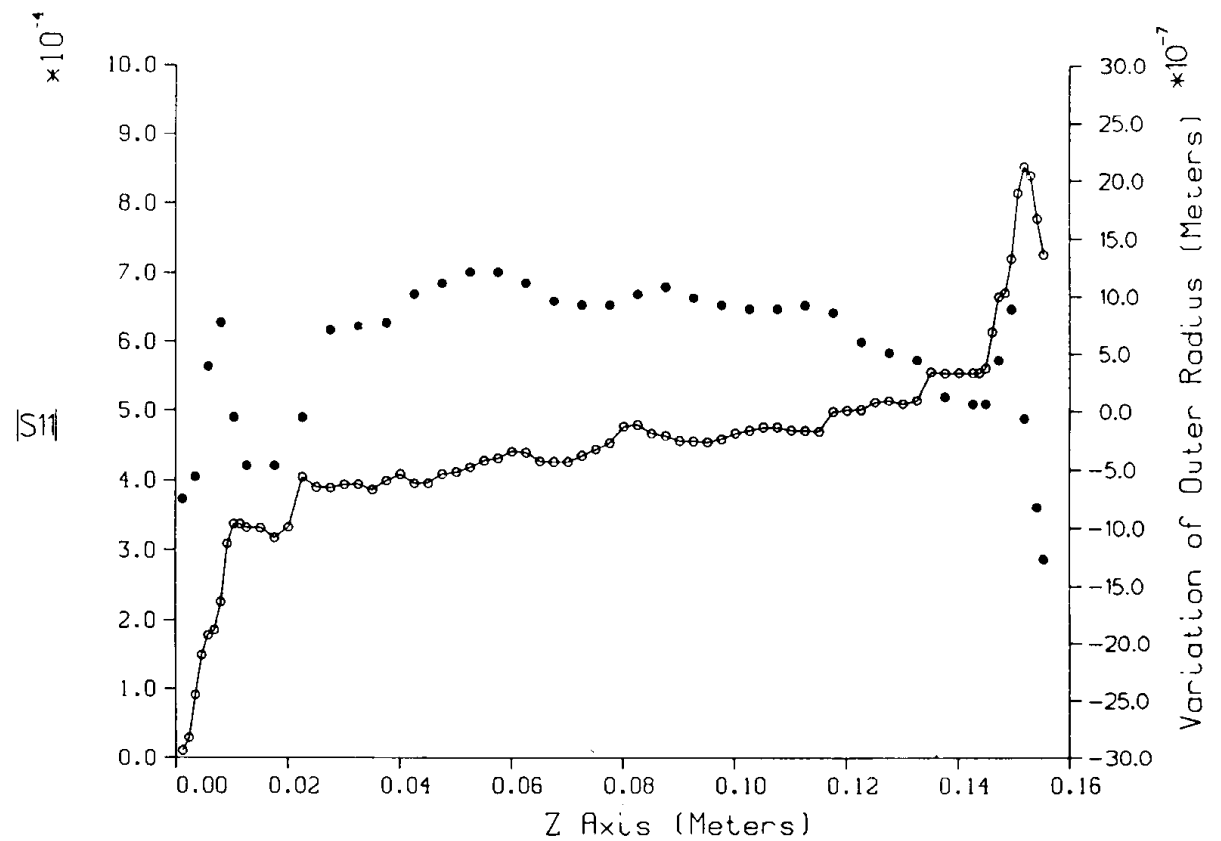

Fig. 10. Profile of the outer radius of a coaxial line and magnitude of the reflection coefficient for a frequency of $18 \mathrm{GHz}$ ( $7 \mathrm{~mm}$ air line),

profile $\Delta_{o}(z)$ of the line is shown in each case. (The profile is indicated by solid circles and the reflection coefficient by open circles.) There is a small increase in $\left|S_{11}\right|$ with frequency, but it is much less than the linear increase in the upper bound as shown in Fig. 7. Its also interesting that $\left|S_{11}\right|$ does not increase as rapidly with line length as indicated by the upper bound expression in (35). The actual $\left|S_{11}\right|$ stays well below the upper bound expression in (35) for all lengths and frequencies.

\section{APPENDIX A}

\section{Higher ORDER MODES}

When only the $m$ th mode is propagating and all higher order modes are below cutoff, the propagation constant $\beta_{i}^{(0)}$ becomes pure imaginary:

$$
\beta_{i}^{(0)}=-j \Gamma_{i}^{(0)}, \quad i \neq m,
$$

where $\Gamma_{i}^{(0)}$ is the attenuation constant and is pure real. If we substitute (A1) into (6), we get the following expression for the amplitudes of the reflected higher order modes:

$$
A_{i}^{-(1)}(0)=A_{0} \int_{0}^{L} S_{i m}^{-} e^{-\left(j \beta_{m}^{(0)}+\Gamma_{i}^{(0)}\right) z} d z, \quad i \neq m .
$$

From (A2) we can develop the following upper bound:

$$
\left|A_{i}^{-(1)}(0)\right| \leqslant\left|A_{0}\right| \mid S_{i m}^{-}\left[1-e^{-\Gamma_{i}^{(0)} L}\right] / \Gamma_{i}^{(0)} .
$$

The coupling coefficient $S_{i m}^{-}$actually depends on $z$, and by $\left|S_{i m}^{-}\right|$in (A3) we mean the maximum value. For modes well below cutoff, $\Gamma_{i}^{(0)} L$ is normally large, and (A3) reduces to

$$
\left|A_{i}^{-(1)}(0)\right| \leqslant\left|A_{0}\right|\left|S_{i m}^{-}\right| / \Gamma_{i}^{(0)} \text {. }
$$

Thus $\left|A_{i}^{-(1)}(0)\right|$ is inversely proportional to $\Gamma_{i}^{(0)}$ and is not proportional to $L$. This results from the exponential decay which allows only a small portion of the guide (approxi- mately equal to $\left.1 / \Gamma_{i}^{(0)}\right)$ to contribute to the integral. Consequently the amplitudes of the reflected higher order modes are much smaller than that of the reflected dominant mode which is proportional to $L$ as shown by (19). For a more precise comparison, we would need to evaluate the coupling coefficient $S_{i m}^{-}$, but it is of the same order as $S_{m m}^{-}$.

To examine the transmitted higher order modes, we substitute (A1) into (6) and obtain the following expression:

$$
A_{i}^{+(1)}(L)=A_{0} \int_{0}^{L} S_{i m}^{+} e^{-\left[j \beta_{m}^{(0)} z+\Gamma_{i}^{(0)}(L-z)\right]} d z .
$$

From (A5) we can obtain the following upper bound:

$$
\left|A_{i}^{+(1)}(L)\right| \leqslant\left|A_{0}\right|\left|S_{i m}^{+}\right| / \Gamma_{i}^{(0)} .
$$

Thus the upper bound for the transmitted higher order modes has the same form as (A4) except that the forward coupling coefficient $S_{i m}^{+}$appears in place of the backward coupling coefficient. Both coupling coefficients are small first-order quantities proportional to the guide nonuniformity [2].

\section{APPENDIX B \\ Transmission COEFFicient $S_{21}$}

From (7) the transmission coefficient for the dominant $\mathrm{TE}_{10}$ mode of a rectangular waveguide is

$$
S_{21}=e^{-j \beta_{[10]}^{(0)} L}\left[1-j \int_{0}^{L}\left(\beta_{[10]}^{(1)}-\beta_{[10]}^{(0)}\right) d z\right]
$$

The zero-order propagation constant $\beta_{[10]}^{(0)}$ is given by (10), and the first-order propagation constant can be obtained 
from a Taylor expansion:

$$
\beta_{[10]}^{(1)}=\left.\beta_{[10\}}^{(0)}\right|_{a=a_{0}}+\left.\Delta \frac{d \beta_{[10]}}{d a}\right|_{a=a_{0}}
$$

where

$$
\left.\frac{d \beta_{\lfloor 10\rfloor}}{d b}\right|_{a=a_{0}}=\frac{\left(\pi / a_{0}\right)^{2}}{a_{0}\left[k^{2}-\left(\pi / a_{0}\right)^{2}\right]^{1 / 2}} .
$$

If we substitute (B2) into (B3), we obtain

$$
S_{21}=e^{--\lambda \beta_{10191}^{(0)} l} \cdot\left(1-j \delta_{21}\right)
$$

where

$$
\delta_{21}=\frac{\left\langle\Delta_{a}\right\rangle L\left(\pi / a_{0}\right)^{2}}{a_{0}\left[k^{2}-\left(\pi / a_{0}\right)^{2}\right]^{1 / 2}}
$$

and

$$
\left\langle\Delta_{a}\right\rangle=\frac{1}{L} \int_{0}^{L} \Delta_{a}(z) d z
$$

Thus $\delta_{21}$ is proportional to the average value of $\Delta_{a}$ and is normally much less than one. Also, $\delta_{21}$ is independent of $\Delta_{b}$.

For the usual case of $\left|\delta_{21}\right| \ll 1$, we can write $S_{21}$ in the following exponential form:

$$
S_{21}=e^{-i\left(\beta_{\mid 1(1)}^{(1)} l+\delta_{21}\right)} .
$$

Thus to first order the magnitude of $S_{21}$ is one, and $S_{21}$ undergoes an additional phase shift of $-\delta_{21}$.

For the coaxial line, the propagation constant $\beta_{0}$ of the TEM mode is equal to $k$ and is independent of $z$. Consequently, the transmission coefficient $S_{21}$ is the same as that of the uniform line to first order:

$$
S_{21}=e^{-j k l} \text {. }
$$

\section{APPENDIX C IMPERFECTLY CONDUCTING WaLlS}

For imperfect wall conductivity, the analysis is in general much more complicated, and even the modes for the uniform waveguide are difficult to analyze [10]. The analysis for the coupling coefficients for nonuniform waveguides is very complicated, but the simpler two-dimensional case of a parallel-plate waveguide has been analyzed using the surface-impedance boundary condition [11].

For metal waveguides of high conductivity, the surface impedance is very small, and the mode fields do not differ much from those of the perfectly conducting waveguide. Consequently the coupling coefficients for nonuniform waveguides which depend on the mode fields do not differ much from those of perfectly conducting waveguides. The main effect of imperfect conductivity is to cause attenuation and a small change in the phase constant [9]. For the rectangular waveguide, the complex propagation constant, $\gamma=\alpha+j \beta$, of the $\mathrm{TE}_{10}$ mode is approximately [10, p. 193]

$$
\gamma \approx j \beta_{[10]}^{(0)}\left\{1+\frac{2 Z_{w}}{j \eta_{0} k_{0} a_{0} b_{0}}\left[a_{0}+\left(\frac{\pi}{a_{0}}\right)^{2} \frac{2 b_{0}+a_{0}}{\beta_{[10]}^{(0) 2}}\right]\right\}^{1 / 2}
$$

where $\eta_{0}$ is the free-space impedance, $k_{0}$ is the free-space wavenumber, and $Z_{w}$ is the surface impedance of the waveguide walls. For high wall conductivity, the surface impedance is

$$
Z_{w}=\left(j \omega \mu_{n} / \sigma_{w}\right)^{1 / 2}
$$

where $\mu_{n}$ is the wall permeability and $\sigma_{n}$ is the wall conductivity.

To account approximately for the effects of finite wall conductivity, we can replace $j \beta_{[10]}^{(0)}$ in Section III by $\gamma$ as given by $(\mathrm{C} 1)$. In most cases this effect will be negligible. For example [9], a copper waveguide at $X$ band has an attenuation rate $\alpha$ of approximately $10^{-2} \mathrm{~Np} / \mathrm{m}$ and a relative change in the real part of $\beta_{\{10]}^{(0)}$ of less than $10^{-3}$. If we make the $\gamma$ substitution for $j \beta_{[10]}^{(0)}$ in (23) and (24), the relative changes in $\left|S_{11 a}\right|$ and $\left|S_{11 b}\right|$ in Figs. 4 and 5 are less than $10^{-3}$.

The correction for finite wall conductivity can be made in a similar manner for the coaxial line. Here the complex propagation constant, $\gamma=\alpha+j \beta$, of the TEM mode is approximately [12]

$$
\gamma \approx j k_{0}+\frac{Z_{n^{\prime}}\left(\rho_{i}{ }^{1}+\rho_{o}{ }^{1}\right)}{2 \eta_{0} \ln \left(\rho_{o} / \rho_{i}\right)}
$$

where $Z_{w}$, is again given by (C2). To account approximately for the effects of finite wall conductivity, we can replace $j k$ in Section IV by $\gamma$ as given by (C3). In most short line applications, the effect will be negligible.

\section{ACKNOWLEDGMENT}

The author would like to thank D. R. Holt for assistance in generating the numerical results and B. C. Yates for useful discussions and comments on the manuscript.

\section{REFERENCES}

[1] A. F. Stevenson, "General theory of electromagnetic horns," $J$ Appl. Phys., vol. 22, pp. 1447-1454, 1951

[2] L. Solymar, "Spurious mode generation in nonuniform waveguide," IEEE Trans. Microwave Theory Tech., vol. MTT-7, pp. 379-383, 1959.

[3] S. A. Schelkunoff, "Conversion of Maxwell's equations into generalized telegraphist's equations," Bell Sist. Tech. J., vol. 34. pp. 995-1044, 1955.

[4] G. Reiter, "Generalized telegraphist's equation for waveguides of varying cross-section," Proc. Inst. Elec. Eng., vol. 106. Pt. B, Supp. 13 , pp. $54-57,1959$.

[5] C. A Hoer and G. F. Engen, "Calibrating a dual six-port or four-port for measuring two-ports with any connections," in 1986 IEEE MTT-S Int. Microwave S!mp. Dig. (Baltimore, MD), June 2-4, 1986, pp. 665-668

[6] D. E. Barrick, "First-order theory and analysis of MF/HF/NHF scatter from the sea," IEEE Trans. Antennas Propagat., vol. AP-20, pp. 2-10, 1972.

[7] B. C. Yates, National Bureau of Standards, Boulder, $\mathrm{CO}$, private communication, 1987.

[8] P. I. Kuznetzov and R. L. Stratonovich, The Propagation of Electro magnetic Waves in Multiconductor Transmission lines. New York: Pergamon, 1964, ch. 5.

[9] E. J. Griffin and T. E. Hodgetts. "Effect on return loss of dimensional tolerances of the aperture of rectangular waveguide," Electron. Lett., vol. 21, pp. 942-943, 1985. 
[10] R. E. Collin, Field Theory of Guided Waves. New York: McGraw-Hill, 1960 , ch. 5.

[11] R. L. Gallawa, "Propagation in nonuniform waveguides with impedance walls," J. Res. NBS, vol. 68D, pp. 1201-1213, 1964.

[12] J. A. Stratton, Electromagnetic Theory. New York: McGraw-Hill, 1941, Sec. 9.21.

世

David A. Hill (M'72-SM'76-F'87) was born in Cleveland, OH, on April 21,1942 . He received the B.S.E.E. and M.S.E.E degrees from Ohio University, Athens, in 1964 and 1966, respectively, and the Ph.D. degree in electrical engineering from Ohio State University, Columbus, in 1970.

Since 1970 he has been a member of the Boulder, CO, scientific community. From 1970 to 1971 he was a Visiting Fellow with the Cooperative Institute for Research in Environmental Sciences where he worked on pulse propagation. From 1971 to 1982 he was with the Institute for Telecommunication Sciences where he worked on theoretical problems in antennas and propagation. Since 1982 he has been in the Electromagnetic Fields Division of the National Bureau of Standards where he has been working on EMC/EMI problems. He is also a Professor Adjoint in the Department of Electrical and Computer Engineering of the University of Colorado.

Dr. Hill is a member of URSI Commissions B, E, and F and a Fellow of IEEE. He has served as a Technical Editor for the IEEE TRANSACIIONS ON GEOSCIENCE AND REMOTE SENSING and is now an Associate Editor for the IEEE Transactions on Antennas and Propagation. 